\title{
Fasting and postprandial levels of ghrelin, leptin and insulin in lean, obese and anorexic subjects
}

\author{
Emilia Korek, Hanna Krauss, Magdalena Gibas-Dorna, Justyna Kupsz, Małgorzata Piątek, Jacek Piątek \\ Department of Physiology, Poznan University of Medical Sciences, Poznan, Poland
}

Prz Gastroenterol 2013; 8 (6): 383-389

DOI: $10.5114 / p g .2013 .39922$

Key words: ghrelin, leptin, insulin, obesity, anorexia nervosa.

Address for correspondence: Emilia Korek MD, PhD, Department of Physiology, Poznan University of Medical Sciences, 6 Swiecickiego St 60-781 Poznan, Poland, phone: +48 6185465 40, fax: + 486185465 39, e-mail: emiliakorek@interia.pl

\begin{abstract}
Introduction: Ghrelin, leptin and insulin are involved in neurohormonal regulation of energetic homeostasis.

Aim: We investigated the correlation between nutritional status and plasma levels of leptin, ghrelin and insulin in lean, obese and anorexic subjects.

Material and methods: Nineteen obese and 18 anorexic adults were enrolled in the study. Seventeen adults with normal body mass index (BMI) served as controls. Blood samples were taken twice: before breakfast and $2 \mathrm{~h}$ after breakfast. Fasting and postprandial ghrelin, leptin and insulin were examined. The following correlations were estimated: between BMI and basal level of tested hormones, between insulin and ghrelin, and between insulin and leptin. The threshold level of significance was $p \leq 0.05$ for all calculations.

Results: Basal insulin level was lowest in anorexic patients and greatest in obese subjects. Fasting plasma ghrelin was lower in obesity and higher in anorexia as compared with the controls. Comparing with controls, fasting leptin levels were higher in obese and lower in anorexic subjects. There was positive correlation between BMI and basal leptin level in obesity. A significant postprandial increase was noted for insulin in all studied groups. Increased leptin and decreased ghrelin levels were detected $2 \mathrm{~h}$ after a meal in the control group. In obese patients, postprandial leptin was lower than before food intake, and fasting leptin showed positive correlation with basal insulin level.

Conclusions: Basal plasma ghrelin, leptin and insulin levels differ according to nutritional status. Impaired ghrelin and leptin secretion and insulin sensitivity may be involved in the pathogenesis of eating disorders.
\end{abstract}

\section{Introduction}

Afferent signals to the brain associated with food intake are transmitted mainly by the "three fat hormones" ghrelin, leptin and insulin. Ghrelin and leptin exhibit opposing effects on energy homeostasis. Ghrelin, secreted by the stomach directly before a meal, acts as a potent and fast appetite stimulant and affects positive energy balance by decreasing glucose and fat oxidation and increasing energy storage [1]. In contrast to ghrelin, leptin promotes suppression of appetite and increased energy use and is associated with long-term energy balance [2]. Leptin is mostly secreted by adipocytes. Its plasma level is influenced by the amount of body fat and correlates in proportion to adiposity. Both ghrelin and leptin affect insulin sensitivity and glucose homeostasis [3], while insulin plays a role in regulating body weight through its down-regulating effects on plasma ghrelin concentrations [4].
Since a growing number of populations suffer from obesity and other eating disorders, studies on determining the role of neurohormonal regulation of food intake and energy balance appear to be of particular importance. Many experimental and clinical trials have shown that impaired ghrelin/leptin/insulin secretion and sensitivity may be involved in the pathogenesis of eating disorders. However, the exact mechanisms of these interactions are still not well understood and some of the reports show contradictory results. For that reason, we have investigated the correlation between nutritional status and plasma levels of leptin, ghrelin and insulin in obese subjects and patients with anorexia nervosa.

\section{Material and methods}

Study participants

Nineteen obese patients ( 9 women and 10 men, aged from 20 to 35 years, mean body mass index (BMI) 
$\left.34.7 \pm 4.92 \mathrm{~kg} / \mathrm{m}^{2}\right)$ and 18 patients with diagnosed anorexia nervosa (18 women aged from 20 to 35 years, mean BMI $16.5 \pm 0.7 \mathrm{~kg} / \mathrm{m}^{2}$ ) were included in this study. The control group comprised seventeen healthy individuals ( 8 women and 9 men) aged from 19 to 25 years with mean BMI $21.1 \pm 1.85 \mathrm{~kg} / \mathrm{m}^{2}$.

All the patients gave their written consent for participation in the study, and the study was conducted with the approval of the Ethical Committee of Poznan University of Medical Sciences.

\section{Study design}

To determine fasting and postprandial ghrelin, leptin and insulin levels, blood samples were collected twice - before breakfast (after 12-h starvation) and $2 \mathrm{~h}$ after food intake. All participants had the same breakfast. The energetic value of the meal was approximately $260 \mathrm{kcal}$ and it contained $4.7 \mathrm{~g}$ of proteins, $14.2 \mathrm{~g}$ of fats and $28.2 \mathrm{~g}$ of carbohydrates (including $14.3 \mathrm{~g}$ of sugar). Blood samples were put into tubes containing potassium EDTA (50 $\mu \mathrm{l}$ liquid/ml blood), centrifuged (10 $\mathrm{min}, 5,000 \mathrm{rpm}, 4^{\circ} \mathrm{C}$ ) and the supernatant plasma was separated for further storage at $-20^{\circ} \mathrm{C}$. Plasma concentrations of tested hormones were estimated using commercially available assays:

1. Plasma concentration of active ghrelin was assessed by Ghrelin/Active/Radioimmunoassay/RIA/Kit (No cat. GHRA - 88HK) of LINCO Research (USA).

2. Plasma concentration of total ghrelin was assessed by Ghrelin/Total/Radioimmunoassay/RIA/Kit (No cat. GHRT - 89HK) of Millipore (USA).

3. Plasma concentration of leptin was measured by Enzyme Linked Immunosorbent Assay (ELISA) on the strength of double binding test (Sandwich ELISA), using DRG Leptin ELISA Kit (No cat. EIA-2395) of DRH Instruments $\mathrm{GmbH}$ (Germany).

4. Plasma insulin concentration was assessed by Enzyme Linked Immunosorbent Assay (ELISA) on the strength of double binding test (Sandwich ELISA), using DRG Insulin ELISA Kit (No cat. EIA-2935) of DRG Instruments $\mathrm{GmbH}$ (Germany).

\section{Statistical analysis}

All statistical analyses were performed using Statistica statistical package program version 9 . Values are expressed as means \pm standard deviation. Statistical comparisons between the two groups were performed using $T$-test for independent trials. To compare values before and after the meal within one group, the $T$-test for dependent trials was used. The correlation of parameters was determined using Pearson's linear correlation ratio. The threshold level of significance was $p \leq 0.05$.

\section{Results}

Fasting ghrelin and leptin levels in the control group, in obese subjects and in anorexic patients

Mean plasma concentration of fasting active ghrelin in obese subjects (OS) was $19.06 \pm 4.85 \mathrm{pg} / \mathrm{ml}$ and was significantly lower than in the control group $(63.38 \pm 23.12 \mathrm{pg} / \mathrm{ml}, p \leq 0.05)$. The level of fasting active ghrelin in patients with anorexia nervosa (77.03 $\pm 3.57 \mathrm{pg} / \mathrm{ml}$ ) was significantly higher when compared with controls $(p \leq 0.05)$. In comparison with the control group, fasting total ghrelin was lower in OS and higher in anorexic patients (ANP) (1625.66 $\pm 450.64 \mathrm{pg} / \mathrm{ml}$ vs. $661.09 \pm 270.57 \mathrm{pg} / \mathrm{ml}$, and 1625.66 $\pm 450.64 \mathrm{pg} / \mathrm{ml}$ vs. $1950.53 \pm 179.69 \mathrm{pg} / \mathrm{ml}$, respectively; $p \leq 0.05)$. Fasting leptin plasma concentration in OS $(42.28 \pm 16.83 \mathrm{ng} / \mathrm{ml})$ and in ANP $(3.29 \pm 1.23 \mathrm{ng} / \mathrm{ml})$ differed significantly when compared with controls (10.10 $\pm 4.64 \mathrm{ng} / \mathrm{ml})(p \leq 0.05)$.

\section{Analysis of correlation between body}

mass index and fasting plasma

concentration of ghrelin and leptin

in obese subjects and anorexic patients

Analysis of reciprocal correlations between ghrelin plasma concentration and BMI demonstrated that active and total ghrelin in OS negatively correlated with $\mathrm{BMI}$, but these dependences were not statistically significant. Similarly in ANP, active and total ghrelin plasma concentrations negatively correlated with BMI, and these correlations were not statistically significant (Table I)

Fasting leptin plasma concentration in OS positively and significantly correlated with BMI (Figure 1). In ANP, positive correlation between leptin and BMI was observed, but it was not statistically significant.

\section{Comparison between fasting}

and postprandial concentrations

of ghrelin, leptin and insulin within

the tested groups (obese subjects, anorexic patients and controls)

Plasma concentrations of active and total ghrelin dropped in the control group after food intake. The difference between fasting and postprandial total ghrelin was not statistically significant. In OS we observed decreases in both active and total ghrelin levels during the $2 \mathrm{~h}$ following the meal. Neither of these changes was statistically significant. In ANP, total and active ghrelin plasma concentrations did not show any statistical significance when comparing fasting with postprandial values. We noted a statistically significant increase of 
Table I. Correlations between BMI and plasma concentrations of ghrelin (active and total) and leptin in obese subjects and patients with anorexia nervosa

\begin{tabular}{lccc} 
Correlation & \multicolumn{1}{c}{$\begin{array}{c}\text { Obese subjects } \\
(n=19)\end{array}$} & \multicolumn{1}{c}{$\begin{array}{c}\text { Subjects with anorexia nervosa } \\
(n=18)\end{array}$} & $r$ \\
\cline { 2 - 4 } & $r$ & 0.64 & -0.19 \\
\hline BMI vs. active ghrelin & -0.11 & 0.72 & -0.05 \\
\hline BMI vs. total ghrelin & & & 0.43 \\
\hline BMI vs. leptin & -0.09 & & 0.84 \\
\hline
\end{tabular}

Values are expressed as mean $\pm S D$. The threshold level of significance is $p \leq 0.05$

Table II. Comparison of mean values of fasting and postprandial ( $2 \mathrm{~h}$ after the meal) plasma concentrations of ghrelin, leptin and insulin in control, obese and anorexic subjects

\begin{tabular}{|c|c|c|c|c|c|c|c|c|c|}
\hline \multirow[t]{2}{*}{ Parameter } & \multicolumn{3}{|c|}{$\begin{array}{l}\text { Control group } \\
(n=17)\end{array}$} & \multicolumn{3}{|c|}{$\begin{array}{l}\text { Obese subjects } \\
(n=19)\end{array}$} & \multicolumn{3}{|c|}{$\begin{array}{l}\text { Subjects with anorexia } \\
\text { nervosa }(n=18)\end{array}$} \\
\hline & Before meal & $\begin{array}{l}\text { After } \\
\text { meal }\end{array}$ & $\begin{array}{l}\text { Value } \\
\text { of } p\end{array}$ & $\begin{array}{l}\text { Before } \\
\text { meal }\end{array}$ & $\begin{array}{l}\text { After } \\
\text { meal }\end{array}$ & $\begin{array}{l}\text { Value } \\
\text { of } p\end{array}$ & $\begin{array}{l}\text { Before } \\
\text { meal }\end{array}$ & $\begin{array}{l}\text { After } \\
\text { meal }\end{array}$ & $\begin{array}{l}\text { Value } \\
\text { of } p\end{array}$ \\
\hline $\begin{array}{l}\text { Active ghrelin } \\
{[\mathrm{pg} / \mathrm{ml}]}\end{array}$ & $63.38 \pm 23.12$ & $54.15 \pm 17.38$ & 0.93 & $19.06 \pm 4.85$ & $18.81 \pm 6.77$ & 0.87 & $77.03 \pm 3.57$ & $79.68 \pm 3.10$ & 0.52 \\
\hline $\begin{array}{l}\text { Total ghrelin } \\
{[\mathrm{pg} / \mathrm{ml}]}\end{array}$ & $\begin{array}{l}1625.66 \\
\pm 450.64 \\
\end{array}$ & $\begin{array}{l}1537.27 \\
\pm 451.88 \\
\end{array}$ & 0.51 & $\begin{array}{r}661.09 \\
\pm 270.57 \\
\end{array}$ & $\begin{array}{r}650.56 \\
\pm 248.53 \\
\end{array}$ & 0.37 & $\begin{array}{r}1950.53 \\
\pm 179.69 \\
\end{array}$ & $\begin{array}{l}1998.22 \\
\pm 194.35 \\
\end{array}$ & 0.45 \\
\hline Leptin [ng/ml] & $10.10 \pm 4.64$ & $18.59 \pm 4.18^{\star}$ & $\leq 0.05$ & $42.28 \pm 16.83$ & $38.08 \pm 13.71^{*}$ & $\leq 0.05$ & $3.29 \pm 1.23$ & $3.01 \pm 0.95$ & 0.50 \\
\hline Insulin $[\mu \mid \mathrm{U} / \mathrm{ml}]$ & $6.93 \pm 2.10$ & $10.69 \pm 3.38^{*}$ & $\leq 0.05$ & $11.84 \pm 6.69$ & $41.95 \pm 16.47^{\star}$ & $\leq 0.05$ & $3.86 \pm 0.59$ & $7.03 \pm 1.63^{*}$ & $\leq 0.05$ \\
\hline
\end{tabular}

Values are expressed as mean \pm standard deviation. The threshold level of significance is $p \leq 0.05$

postprandial leptin in the controls and a decrease in OS, when compared with preprandial values. In ANP, postprandial leptin did not differ significantly. In comparison with fasting levels, postprandial plasma concentrations of insulin were elevated in all three groups ( $p \leq 0.05)$. The greatest increase of postprandial insulin concentration (hyperinsulinaemia) was observed in OS (Table II).

\section{Analysis of the correlation between fasting insulin plasma concentrations and fasting plasma concentrations of ghrelin and leptin in subjects with obesity, anorexia nervosa and normal body mass index}

Positive correlation was found between fasting total ghrelin plasma concentration and fasting insulin plasma concentration in OS, but the relationship was not statistically significant $(r=0.07, p=0.78)$. In ANP, negative correlation between fasting total ghrelin plasma concentration and fasting insulin plasma concentration was observed, but this relationship was also not statistically significant $(r=-0.16, p=0.52)$. Significant correlation was not present between fasting total ghrelin and fasting insulin plasma concentration in controls $(r=0.13, p=0.62)$.

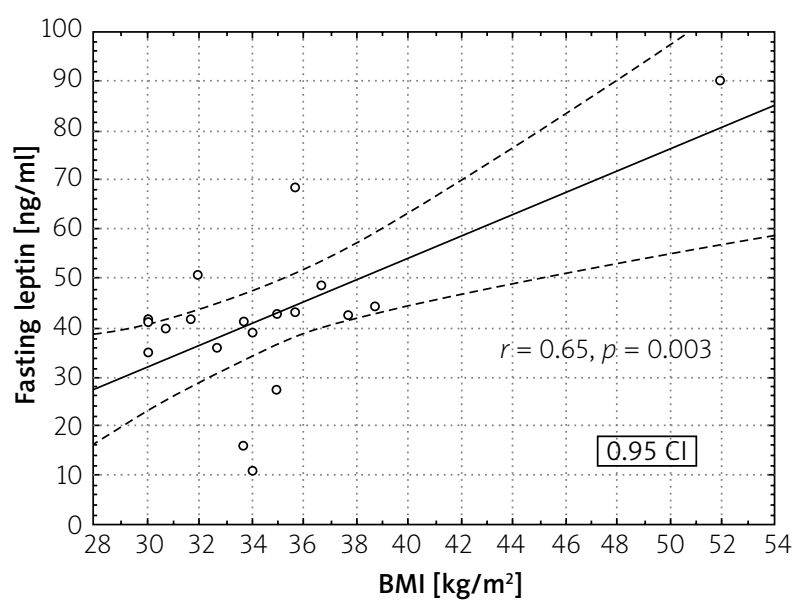

Figure 1. Correlation between fasting leptin concentrations and $\mathrm{BMI}$ in obese subjects

Positive and statistically significant correlation between fasting leptin and fasting insulin plasma concentration was present in the control group $(r=0.48$, $p=0.05$ ) (Figure 2).

Positive and statistically significant correlation between fasting leptin and fasting insulin plasma concen- 


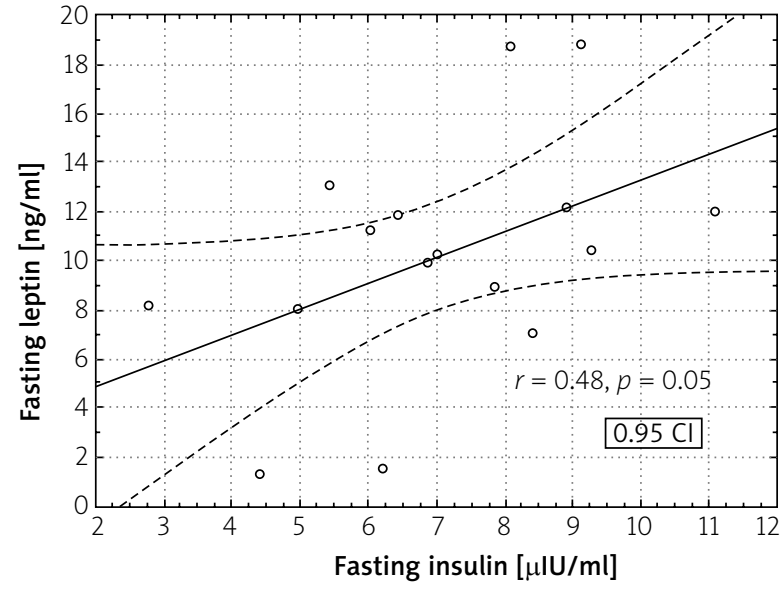

Figure 2. Correlation between fasting leptin and fasting insulin plasma concentrations in control group

tration was also found in OS ( $r=0.53, p=0.02)$ (Figure 3). Negative correlation between fasting leptin and fasting insulin plasma concentration in ANP was present, but it was not statistically significant $(r=-0.18, p=0.46)$.

\section{Discussion}

It has been consistently reported that obesity and eating disorders, which affect the health of people worldwide and sometimes are life threatening, are closely related to certain peripheral metabolic factors, including leptin, ghrelin and insulin. Despite intensive research the exact mechanisms of neuroendocrine regulation of food intake and energy balance are still unclear. Therefore, our results summarizing the levels of leptin, ghrelin and insulin in obese and anorexic patients serve as an additional source of knowledge.

\section{Ghrelin in obesity}

The low level of ghrelin in obesity is thought to be part of the compensatory mechanism to the prolonged positive energy balance, since ghrelin works as an indicator of energy insufficiency and a hunger stimulator in the organism. Our findings demonstrate that obesity is associated with lowered basal plasma ghrelin, which confirms several previously published observations reporting that suppression of ghrelin release is related to obesity, and that fasting plasma ghrelin levels are negatively correlated with the percentage of body fat and body weight [5]. Postprandial ghrelin responses in normal-weight humans result in the lowering of its level due to the energy intake. The restoration of postprandial ghrelin response is observed in obese subjects after diet-induced weight loss [6], indicating (a) improved insulin sensitivity as an effect of weight loss and (b) normal inhibition of ghrelin secretion induced by insulin. In

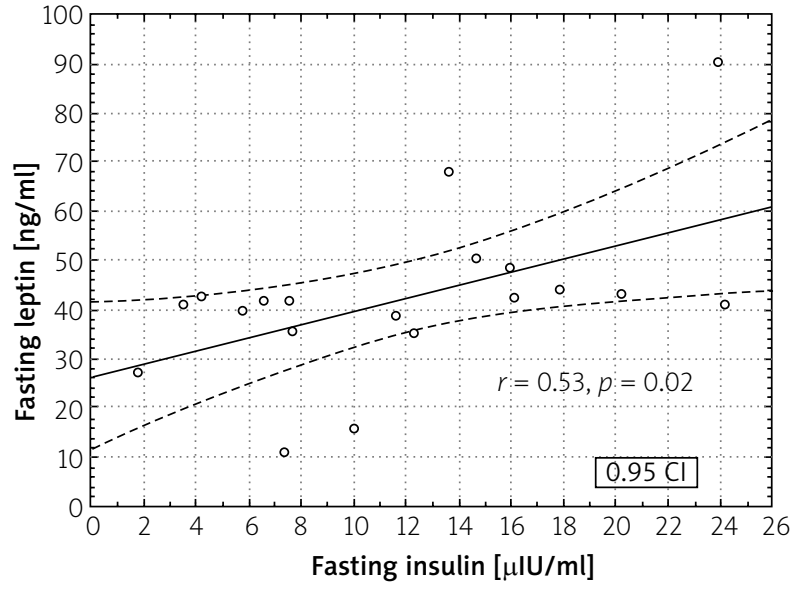

Figure 3. Correlation between fasting leptin and fasting insulin plasma concentrations in obese subjects

untreated obese subjects, however, the reports are less evident. This effect, as reported by other authors, partially depends on meal composition. Fatty meals less effectively suppress ghrelin secretion in comparison with a diet rich in proteins or carbohydrates [7]. According to the concept that insulin is an inhibitor of ghrelin secretion, postprandial suppression of ghrelin is associated with meals containing a sufficient amount of carbohydrate to raise glucose and insulin levels substantially [8]. In our experiment the meal consisted of $50 \%$ fats, $35 \%$ carbohydrates and $15 \%$ proteins. Probably because the breakfast we used was fatty, we noted a tendency towards decreased postprandial ghrelin levels in obese subjects, which was not significant.

\section{Ghrelin in anorexia}

Anorexia nervosa is one of the eating disorders in which fasting plasma ghrelin is markedly elevated and normalizes after weight gain [9]. Regarding the relationship between high ghrelin and low BMI described by other authors, we observed increased basal plasma ghrelin in patients with anorexia nervosa, when compared with the controls. A high ghrelin level is the body's response to negative energy balance and a neuroendocrine signal of body hunger. However, as suggested by Miller, a strong ghrelin resistance in anorexic patients exists [10]. This may be explained by two mechanisms: decrease sensitivity to endogenous ghrelin and/or complex interactions at hypothalamus. As a result, a chronically elevated ghrelin concentration does not prevent progression of the disorder.

Interestingly, in anorexic patients we did not observe any statistically significant change in either postprandial total or postprandial active ghrelin levels. As reported by Murdolo et al., insulin mediates the ef- 
fect of nutrition on postprandial regulation of plasma ghrelin concentrations in humans [11]. We suspect that our anorexic patients demonstrated insensitivity to the suppressive effects of postprandial insulin on plasma ghrelin levels. Our results are contradictory to the reports of Karczewska-Kupczewska et al., who described the presence of increased suppression of serum ghrelin by hyperinsulinaemia in women with anorexia nervosa. However, they examined patients who underwent an oral glucose tolerance test and exhibited high level of hyperinsulinaemia (24.86 $\pm 16.65 \mathrm{mmol} / \mathrm{l})$ in response to pure glucose stimulation [12]. Similarly to our findings, Nedvidková et al. reported that the acute plasma ghrelin response to food intake, which in healthy individuals is independent of meal caloric value, is impaired in women with anorexia [13]. We also speculate a possible effect of meal macronutrient composition on postprandial insulin sensitivity, which, according to the literature, is lower after high-fat meals.

\section{Leptin in obesity}

In our study fasting leptin plasma concentration in obese patients was significantly higher when compared with the controls. These results are consistent with the findings of other authors [14]. In obese people, levels of leptin are normally very high and theoretically more than sufficient to suppress appetite and accelerate metabolism. However, the hyperleptinaemic state of obesity has been associated with leptin resistance and with central leptin deficiency [15]. There are several suggested mechanisms of leptin resistance in obesity: blockade of signalling in the hypothalamus, disturbances of leptin transport through the blood-brain barrier and decreased sensitivity or decreased number of leptin receptors (OB-RO), and disrupted signalling tracks in the hypothalamus [16].

When comparing pre- with postprandial leptin levels we noted that the plasma leptin response pattern differed between obese subjects and the controls, depending on body composition. For the controls we observed an increase in leptin concentration $2 \mathrm{~h}$ after the meal, while in obese patients leptin levels decreased significantly. Our study revealed that the change in leptin levels after a single high-fat meal might occur earlier than reported by other authors [17]. To some extent, our results support the notion that the postprandial leptin response could be a consequence of the diminished effect of insulin on adipocytes, since obesity is strongly related with poor insulin sensitivity. Lowered postprandial leptin in obese patients may possibly contribute to the behavioural encouragement of overeating.

\section{Leptin in anorexia}

Decreased leptin concentrations in patients with negative energy balance are related with adaptive mechanisms to chronic malnutrition and starvation. Anorexic patients have significantly lower leptin levels than normal, which suggests that leptin is not involved in the reduction of food intake associated with anorexia, but really reflects total body fat mass [18]. The low levels progressively rise in anorexic patients undergoing re-feeding treatment, but they do not serve as a direct appetite stimulant in such a disorders, indicating resistance to the deficiency of that hormone.

In our patients with anorexia, fasting leptin plasma concentrations were lower than in subjects with normal $\mathrm{BMI}$ and much lower than the threshold-value (fasting leptin below $10.72 \mathrm{ng} / \mathrm{ml}$ ) described by Ziora et al. [19], which characterizes anorexic patients. Our patients had basal leptin at a mean level of $3.29 \pm 1.23 \mathrm{ng} / \mathrm{ml}$. The presented findings are supported by other reports based on leptin levels in anorexia nervosa [20].

Postprandial levels of leptin did not bring any major changes in anorexic patients. Perhaps the fact that leptin levels are unchanged by ingestion of a single meal, and that they remain low, plays role in the defence mechanism against the anorexic effects of this hormone, or maybe the effects occur later.

\section{Leptin and body mass index}

Our results confirm that plasma fasting leptin positively correlates with BMI and the amount of white adipose tissue, suggesting that nutritional status is an important regulator of plasma leptin levels. As shown by us, and by other authors [14, 21], fasting leptin concentrations positively and significantly correlate with $\mathrm{BMI}$ in obese patients. In patients with anorexia the non-significant positive correlation may be due to the small group of participants in our study. Most investigators who reported a strong positive correlation but their experiments included patients before and after weight gain, focusing rather on the effects of successful re-nutrition/treatment $[19,22]$.

\section{Insulin levels}

Basal insulin reflected the nutritional status of the study participants, being lowest in anorexic patients and greatest in obese subjects. As expected, insulin levels rose after a meal in all three groups, and the greatest rise was observed in obese subjects (we noted a 4-fold increase in insulin levels) leading to hyperinsulinaemia. These findings are consistent with worldwide studies [23]. 
Circulating ghrelin levels are altered in obesity, and chronically low levels of circulating ghrelin are found in obese patients [24]. Although the correlations between basal insulin and ghrelin did not differ statistically in all the studied groups, we noted the lowest level of ghrelin in patients with hyperinsulinaemia, while the highest ghrelin level was found in anorexic patients (with the lowest basal insulin concentration). We agree with other studies suggesting that high levels of insulin are related to suppression of ghrelin release [25]. The mechanism of ghrelin-insulin regulation is like a twoway street: ghrelin is a potent regulator of insulin level capable of reducing glucose-stimulated insulin secretion; on the other hand, high insulin may inhibit ghrelin release [26, 27].

Leptin secretion is regulated by insulin, glucocorticoids and catecholamines [28]. We found strong positive correlation between fasting leptin and fasting insulin concentrations, which confirms that high leptin levels might be directly associated with the hyperinsulinaemia and insulin resistance observed in obese subjects.

Although Misra et al. [29] observed strong positive correlation between plasma leptin concentrations and insulin resistance in anorexic patients, the relationship between low plasma leptin levels and the degree of insulin sensitivity in these patients is still under investigation. We failed to find any correlation between basal plasma leptin and insulin in ANP, and this indicates one of the limitations of our study: we did not determine peripheral insulin resistance, which would have been helpful in confirming our suggestions regarding insulin sensitivity.

Other limitations include the small number of study participants and the fact that the studied hormones were investigated only twice: before the meal and $2 \mathrm{~h}$ after the meal. Increasing the frequency of sampling in different time intervals would definitely bring more interesting findings.

\section{Conclusions}

This is the first report regarding fasting and postprandial levels of ghrelin, leptin and insulin in lean, obese and anorexic subjects. We conclude that lack of the postprandial inhibition of ghrelin secretion and postprandial hypoleptinaemia in obese patients may play a crucial role in the pathophysiology of the obesity. Positive correlation between fasting leptin and insulin levels in obesity may be related to hyperinsulinaemia and insulin resistance. Acute plasma ghrelin response to food intake is impaired in anorexia nervosa.

\section{Acknowledgments}

This research was funded by the Department of Physiology Poznan University of Medical Sciences grant number 502-0101-1251-8404-401.

\section{References}

1. Cummings DE, Purnell JQ, Frayo RS, et al. A preprandial rise in plasma ghrelin levels suggests a role in meal initiation in humans. Diabetes 2001; 50: 1714-9.

2. Wannamethee SG, Tchernova J, Whincup P, et al. Plasma leptin: associations with metabolic, inflammatory and haemostatic risk factors for cardiovascular disease. Atherosclerosis 2007; 191: 418-26.

3. Soni AC, Conroy MB, Mackey RH, et al. Ghrelin, leptin, adiponectin, and insulin levels and concurrent and future weight change in overweight postmenopausal women. Menopause 2011; 18: 296-301.

4. Erdmann J, Lippl F, Wagenpfeil S, et al. Differential association of basal and postprandial plasma ghrelin with leptin, insulin and type 2 diabetes. Diabetes 2005; 54: 1371-8.

5. Tschop M, Weyer C, Tataranni PA, et al. Circulating ghrelin levels are decreased in human obesity. Diabetes 2001; 50: 707-9.

6. Romon M, Gomila S, Hincker P, et al. Influence of weight loss on plasma ghrelin responses to high-fat and high-carbohydrate test meals in obese women. J Clin Endocrinol Metab 2006; 91: 1034-41.

7. Foster-Schubert KE, Overduin J, Prudom CE, et al. Acyl and total ghrelin are suppressed strongly by ingested proteins, weakly by lipids, and biphasically by carbohydrates. J Clin Endocrinol Metab 2008; 93: 1971-9.

8. Erdmann J, Topsch R, Lippl F, et al. Postprandial response of plasma ghrelin levels to various test meals in relation to food intake, plasma insulin and glucose. J Clin Endocrinol Metab 2004; 89: 3048-54.

9. Otto B, Cuntz U, Fruehauf E, et al. Weight gain decreases elevated plasma ghrelin concentrations of patients with anorexia nervosa. Eur J Endocrinol 2001; 145: 669-73.

10. Miller KK. Endocrine dysregulation in anorexia nervosa update. J Clin Endocrinol Metab 2011; 96: 2939-49.

11. Murdolo G, Lucidi P, Di Loreto C, et al. Insulin is required for prandial ghrelin suppression in humans. Diabetes 2003; 52 : 2923-7.

12. Karczewska-Kupczewska M, Straczkowski M, Adamska A, et al. Increased suppression of serum ghrelin concentration by hyperinsulinemia in women with anorexia nervosa. Eur J Endocrinol 2010; 162: 235-9.

13. Nedvidková J, Krykorková I, Barták V, et al. Loss of meal-induced decrease in plasma ghrelin levels in patients with anorexia nervosa. J Clin Endocrinol Metab 2003; 88: 1678-82.

14. Rosická M, Krsek M, Matoulek M, et al. Serum ghrelin levels in obese patients: the relationship to serum leptin levels and soluble leptin receptors levels. Phys Rev 2003; 52: 61-6.

15. Caro JF, Kolaczynski JW, Nyce MR, et al. Decreased cerebrospinal-fluid/serum leptin ratio in obesity: a possible mechanism for leptin resistance. Lancet 1996; 348: 159-61.

16. Pijl H. Leptin resistance. Ned Tijdschr Klin Chem Labgeneesk 2007; 32: 3-8. 
17. Dallongeville J, Hecquet B, Lebel P, et al. Short term response of circulating leptin to feeding and fasting in man: influence of circadian cycle. Int J Obesity 1998; 22: 728-33.

18. Dostálová I, Kopský V, Dušková J, et al. Leptin concentrations in the abdominal subcutaneous adipose tissue of patients with anorexia nervosa assessed by in vivo microdialysis. Regul Pept 2005; 128: 63-8.

19. Ziora K, Oświęcimska J, Świętochowska E, et al. Serum leptin levels in girls with anorexia nervosa [PoLish]. Endokrynol Pediatr 2010; 9: 43-53.

20. Haas V, Onur S, Paul T, et al. Leptin and body weight regulation in patients with anorexia nervosa before and during weight recovery. Am J Clin Nutr 2005; 81: 889-96.

21. Beasley JM, Ange BA, Anderson CA, et al. Characteristics associated with fasting appetite hormones (obestatin, ghrelin, and leptin). Obesity 2009; 17: 349-54.

22. Grinspoon S, Gulick T, Askari H, et al. Serum leptin levels in women with anorexia nervosa. J Clin Endocrinol Metab 1996; 81: 3861-3.

23. Prager R, Wallace P, Olefsky JM. Hyperinsulinemia does not compensate for peripheral insulin resistance in obesity. Diabetes 1987; 36: 327-34.

24. Tschop M, Smiley DL, Heiman ML. Ghrelin induces adiposity in rodents. Nature 2000; 407: 908-13.

25. Flanagan DE, Evans ML, Monsod TP, et al. The influence of insulin on circulating ghrelin. Am J Physiol Endocrinl Metab 2003, 284: E313-6

26. Tong J, Prigeon RL, Davis HW, et al. Ghrelin suppresses glucose-stimulated insulin secretion and deteriorates glucose tolerance in healthy humans. Diabetes 2010; 59: 2145-51.

27. Poykko SM, Kellokoski E, Horkko S, et al. Low plasma ghrelin is associated with insulin resistance, hypertension, and the prevalence of type 2 diabetes. Diabetes 2003; 52: 2546-53.

28. Friedman JM. Modern science versus the stigma of obesity. Nat Med 2004; 10: 563-9.

29. Misra M, Miller KK, Almazan C, et al. Hormonal and body composition predictors of soluble leptin receptor, leptin, and free leptin index in adolescent girls with anorexia nervosa and controls and relation to insulin sensitivity. J Clin Endocrinol Metab 2004; 89: 3486-95.

Received: 28.03.2013

Accepted: 6.05 .2013 